\title{
Commentary on 'Local treatment with a polycarbophil-based cream in postmenopausal women with genitourinary syndrome of menopause'
}

\author{
Abdelmageed Abdelrahman ${ }^{1}$ \\ Received: 2 April 2020 / Accepted: 7 April 2020 / Published online: 21 April 2020 \\ (C) The International Urogynecological Association 2020
}

This was a prospective single-cohort study to compare symptoms of genitourinary syndrome before and after treatment with polycarbophil-based cream in postmenopausal women. Inclusion criteria included women with one or more external genitalia or sexual symptoms and those with one or more urinary symptoms. Women allergic to polycarbophil-based cream could not undergo uroflowmetry, and those treated with a topical oestrogen cream or vaginal moisturizer within 3 months prior to the study were excluded.

Forty-four women diagnosed with genitourinary syndrome between July 2017 and October 2018 were asked to participate in the study, with two women excluded as they declined to participate. Women were asked to apply the topical vaginal moisturizer cream to the vaginal and urethral opening once every 3 days before bedtime for 3 months. The authors collected pre- and posttreatment (at 4 and 12 weeks) data from patients using the following: quality of life and sexual scores of the Thai version if the International Consultation on Incontinence Modular Questionnaire-Lower Urinary Tract Symptoms and uroflowmetry. The authors con-

Abdelmageed Abdelrahman

abdelmageed@hotmail.co.uk

1 Department of Urogynaecology, Liverpool Women's Hospital NHS Foundation Trust, Liverpool, UK cluded that the use of non-hormonal polycarbophilbased cream for the treatment of genitourinary symptoms of the menopause resulted in significant improvements in validated lower urinary tract and sexual quality of life measures.

The authors present an interesting study with a prospective study design and use of validated quality of life questionnaires. A limitation of the study is the sample size of 42 patients. Also of note, comparisons between the use of placebo and standard treatment (hormonal vaginal preparation) were not made. The study focused on urinary and sexual symptoms only rather than other general gynaecological symptoms.

\section{Compliance with ethical standards}

Conflict of interest None.

Publisher's note Springer Nature remains neutral with regard to jurisdictional claims in published maps and institutional affiliations. 